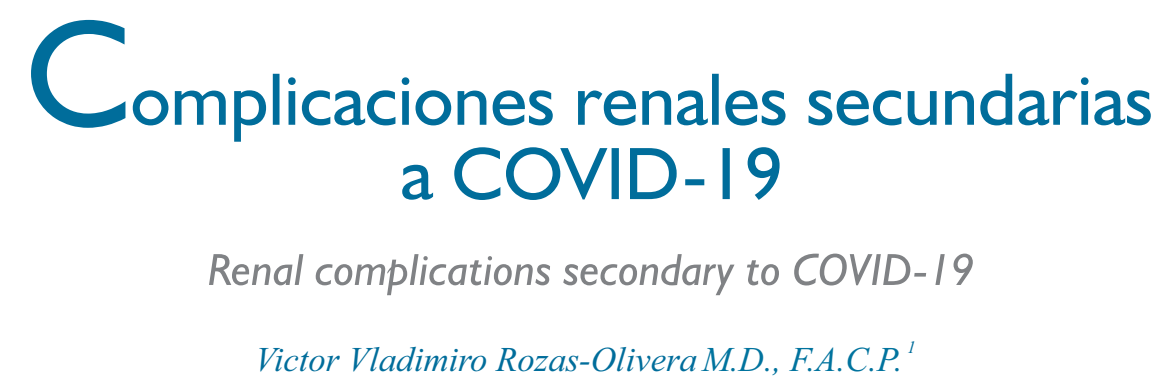

Resumen

Los pacientes tratados con inhibidores o bloqueadores del sistema renina-angiotensina-aldosterona (SRAA) no muestran un riesgo incrementado para la infección con COVID-19 ni de desarrollar un estadio más crítico de la enfermedad. Al momento de ser hospitalizados, los pacientes con COVID-19, en una proporción elevada presentan proteinuria y hematuria. Reportes de Europa y los Estados Unidos muestran que los pacientes con COVID-19, que requieren de cuidados intensivos, presentan una incidencia de injuria renal aguda (IRA) en un porcentaje del $20 \%$ al 40\%; el inicio de la IRA, tiene una relación temporal con el comienzo de la ventilación mecánica. Los hallazgos patológicos en estos pacientes indican la presencia de necrosis tubular aguda como causa principal de la injuria renal. Aproximadamente el $\mathbf{1 4 \%}$ de pacientes con IRA, requiere de terapia de remplazo renal, y están asociados a tasas de mortalidad del 35\% al 55\%. Ante un incremento súbito de pacientes con COVID-19 e IRA, los sistemas de salud necesitaron de esfuerzos extraordinarios para poder proveer personal médico especializado y de equipos para terapia de diálisis.

Palabras clave: COVID-19, sistema renina-angiotensina-aldosterona, injuria renal aguda.

\title{
Abstract
}

Patients treated with inhibitors or blockers of the renin-angiotensin-aldosterone system (RAAS), showed no increased risk to develop COVID-19, nor an increased risk to developed a more critical stage of the disease. Patients with COVID-19, at the time of hospitalization showed a high proportion of the presence of proteinuria and hematuria. Reports from Europe and the United States showed that patients with COVID-19, whom required intensive care unit care, had an incidence of acute kidney injury (AKI) of $20 \%$ to $40 \%$, and its onset showed a temporal relationship to the initiation of mechanical ventilation. Pathological findings showed that the principal cause of the renal injury was the development of acute tubular necrosis. The surge of patients with COVID-19 and AKI, demanded extraordinary efforts from health systems, to provide specialized medical personal and equipment for dialysis therapy.

Keywords: COVID-19, renin-angiotensin-aldosterone system, acute kidney injury.

\section{Virus SARS-CoV-2}

La partícula viral SARS-CoV-2, tiene un diámetro de aproximadamente 100 nanómetros, es visible solo en el microscopio electrónico. Es casi una esfera de proteína cubierta de una membrana de lípidos, la cual protege a la hebra de ARN, la molécula que contiene el genoma viral. Contiene cuatro proteínas: La proteína $\mathrm{S}$, en forma de "espiga" y que le da la apariencia de "corona", la proteína de membrana y la proteína envolvente que le da soporte estructural, y el nucleocápside que actúa como un andamio, rodeada de los 29.900 nucleótidos de ARN que conforman el genoma viral ${ }^{(1)}$.

${ }^{1}$ Miembro del Comité de Educación, Peruvian American Medical Society (PAMS), Profesor Emérito de Medicina Clínica, Universidad del Estado de Michigan, USA, Director Emérito, Great Lakes Renal Network, Michigan, USA. Miembro Correspondiente, Academia Nacional de Medicina del Perú. ORCID ID: 0000-0002-0712-1994 
Role del Sistema Renina-AngiotensinaAldosterona (SRAA)

El virus SARS-CoV-2, se adhiere a las a las células humanas por vía de la enzima convertidora de angiotensina 2 (ECA2), la cual se expresa en las células epiteliales de los pulmones, riñones, tracto intestinal, vasos sanguíneos y corazón $^{(2)}$. La adherencia a la ECA2, constituye la vía principal de acceso del virus al interior celular, donde utiliza la maquinaria celular para reproducirse y seguir potenciando la infección. (Figura 1).

Se ha reportado que la expresión de la ECA2 se encuentra incrementada en pacientes con diabetes e hipertensión, tratados con bloqueadores del sistema reninaangiotensina-aldosterona (SRAA), medicamentos como los inhibidores ECA y los bloqueadores de los receptores de angiotensina (BRA).

Esta expresión aumentada de receptores ECA2, teóricamente podría facilitar la infección y un estadio más severo o crítico de la enfermedad. En la última década, se ha caracterizado un nuevo axis del SRAA: ECA2-Ang- ${ }^{(1-7)}$ Receptor MasR, eje que se opone a los efectos de la vía clásica del SRAA, induciendo efectos de vasodilatación, antiinflamaatorio, anti fibrótico y anti angiogénico, por lo tanto, representa el brazo protector del SRAA, con efectos de protección cardiovascular y renal $^{(3-4)}$.
Se han realizado varios estudios para poder determinar si los bloqueadores del SRAA confieren un incremento de riesgo de infección a COVID-19, o de desarrollar un estadio más severo ${ }^{(5-7)}$. Estos estudios no han demostrado un riesgo incrementado para la infección, ni riesgo incrementado de desarrollar enfermedad más severa o crítica.

Un grupo de investigadores en estudios experimentales en ratones, a los que se les administró un bloqueador del SRAA, no encontraron una mayor expresión de receptores ECA2 a nivel pulmonar, ni a nivel renal ${ }^{(8)}$.

Un estudio reciente realizado en Inglaterra, en el que se hizo un metaanálisis de 28,872 pacientes con COVID-19 para determinar el efecto de inhibidores del SRAA, llegó a la conclusión, que pacientes hipertensos tratados con inhibidores ECA o BRA, mostraron un riesgo menor del 0.67 para tener un resultado fatal o crítico ${ }^{(9)}$.

\section{Fisiopatología y Manifestaciones Clínicas Renales}

La presencia de anormalidades de función renal en pacientes hospitalizados con COVID-19 se hicieron evidentes al ser admitidos al hospital. En pacientes con enfermedad más severa, existe el potencial de compromiso de múltiples órganos, siendo central en la patogénesis el desarrollo de sepsis, inflamación sistémica e hipercoagulabilidad ${ }^{(10)}$. (Figura 2).

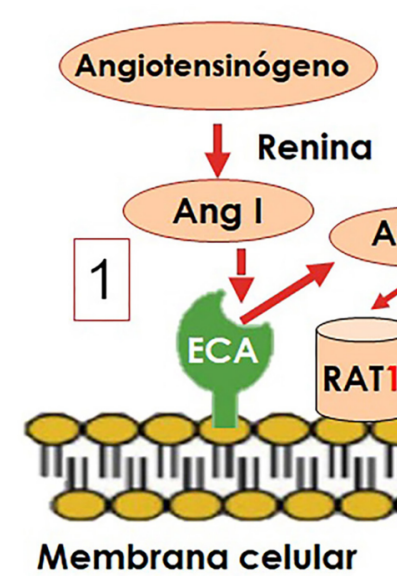

ECA: Enzima convertidora de angiotensina RAT: Receptor de angiotensina TMPRSS2: Proteasa sérica transmembrana 2

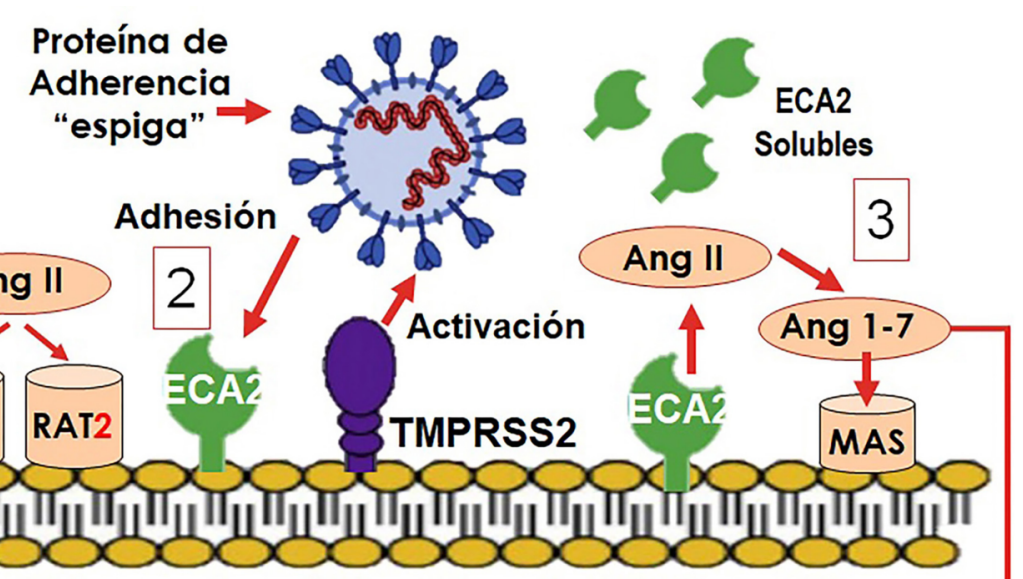

Pulmones, Vasculatura, Corazón, Intestinos y Riñones

Figura 1. Cascada del SRAA. Role de la ECA2 en la adherencia epitelial del SARS-CoV-2. 


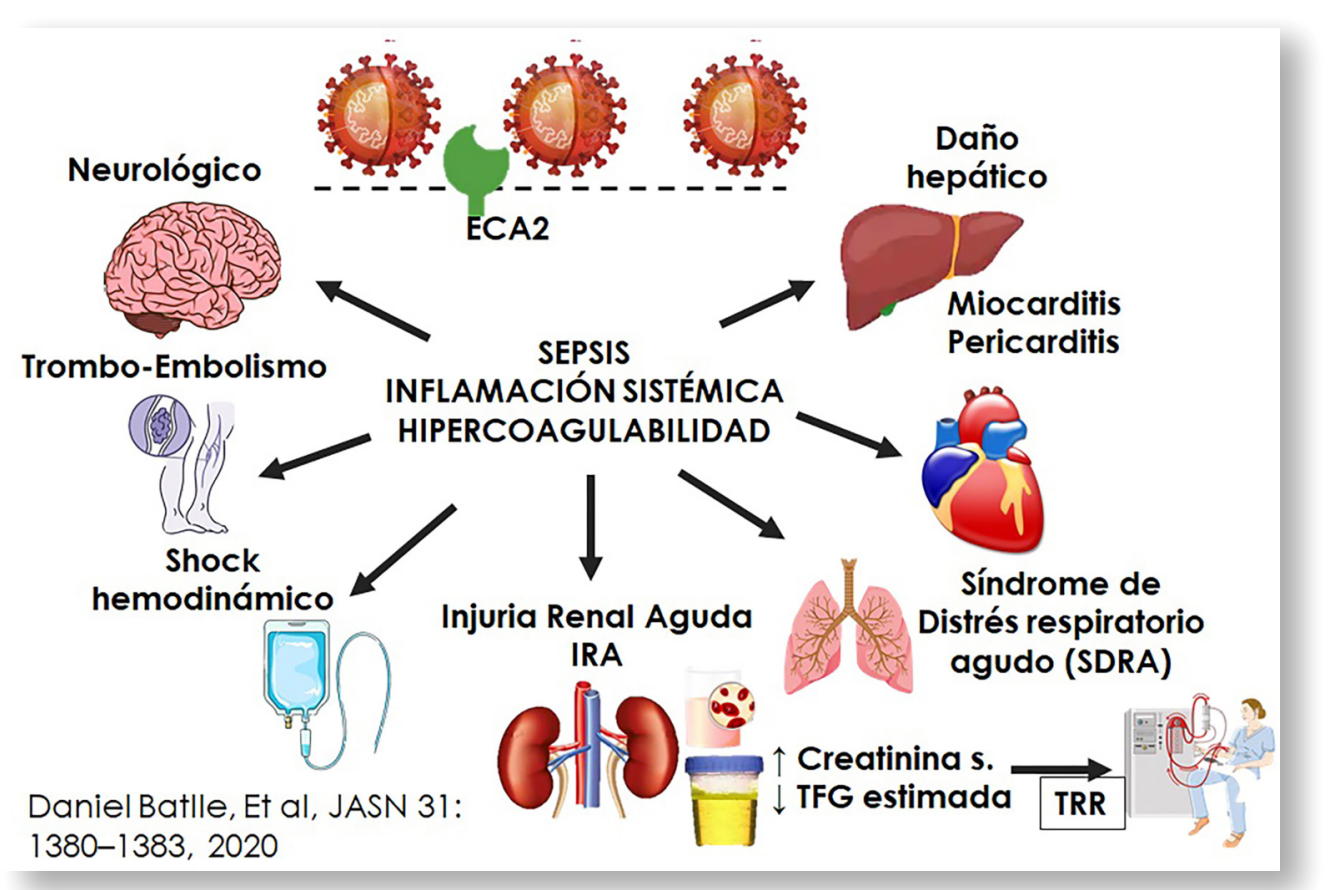

Figura 2. Complicaciones potenciales de múltiples órganos con COVID-19.

Un primer estudio prospectivo de 701 pacientes hospitalizados en Wuhan, China ${ }^{(11)}$, reveló que el $43.9 \%$ presentaron proteinuria y $25.7 \%$ hematuria, de los cuales $10.2 \%$ y $11.3 \%$ respectivamente con niveles más severos $(2+$ a $3+)$. De manera similar un estudio en el Hospital Puerta de Hierro de Madrid, reveló que, al momento de admisión hospitalaria, una quinta parte de los pacientes mostraron creatinina sérica elevada, de los cuales el $40 \%$ de ellos tenían evidencia de enfermedad renal pre existente, $40 \%$ de los pacientes mostraron proteinuria y $50 \%$ hematuria $^{(12)}$. La patogénesis asociada a estas anormalidades aún está por ser definida, ya que no existen reportes histopatológicos en los estadios más tempranos de la enfermedad, se sospecha, sin embargo, que esté asociada a disfunción endotelial ${ }^{(13)}$. Estos hallazgos son diferentes de los que se encuentran asociados a estadios críticos de la enfermedad. Hay cierta analogía a anormalidades renales asociadas a otros tipos de virosis, como es el caso de los virus de inmunodeficiencia, de los virus polioma y hanta, lo que han llevado a proponer el término de Coronavirus Asociada a Nefropatía $\left(\right.$ COVAN) ${ }^{(14)}$. (Figura 3).

En el seguimiento de casos individuales de nefropatía colapsante asociada a pacientes con COVID-19, se ha reportado una serie de seis pacientes con ancestro africano y con COVID-19, con variaciones genéticas del gen APOL1 (apolipoproteína 1). Los pacientes presentaron manifestaciones de injuria renal aguda y proteinuria severa. Los exámenes patológicos renales, mostraron las características de la nefropatía colapsante, con colapso del penacho glomerular, hipertrofia e hiperplasia de las células epiteliales viscerales, así como en microscopía electrónica, se observó desprendimiento extenso de podocitos y su transformación microvellosa ${ }^{(15)}$.

Las variaciones genéticas del gen APOL1 dan un riesgo mayor para desarrollar enfermedades renales y su progresión, lo que explica también la disparidad de la prevalencia de enfermedad renal entre poblaciones de ancestro africano vs europea.

Aproximadamente $5 \%$ de los pacientes con COVID-19 y $20 \%$ de los hospitalizados requieren de cuidados intensivos. Los pacientes son admitidos a unidades de cuidados intensivos debido al desarrollo de hipoxemia e insuficiencia respiratoria y/o la necesidad de utilizar vasopresores, el $91 \%$ de los pacientes requiere de ventilación mecánica y con frecuente compromiso de múltiples órganos vitales: corazón, cerebro, pulmón, hígado, riñón y el sistema de coagulación. Central a la patogénesis está el hecho que el virus SARS-CoV-2 infecta y destruye a los linfocitos $\mathrm{T}$, acoplado a una respuesta inflamatoria viral que consiste de respuesta inmune innata, así como la adaptativa con repuestas humoral y celular ${ }^{(16)}$.

La respuesta inflamatoria está principalmente mediada por la liberación de múltiples citoquinas, ("tormenta de citoquinas"). En forma paralela al incremento de la respuesta inflamatoria, se manifiesta un estado de hipercoagulabilidad con niveles elevados de fibrinógeno y del dímero- $\mathrm{D}^{(17-18)}$. 


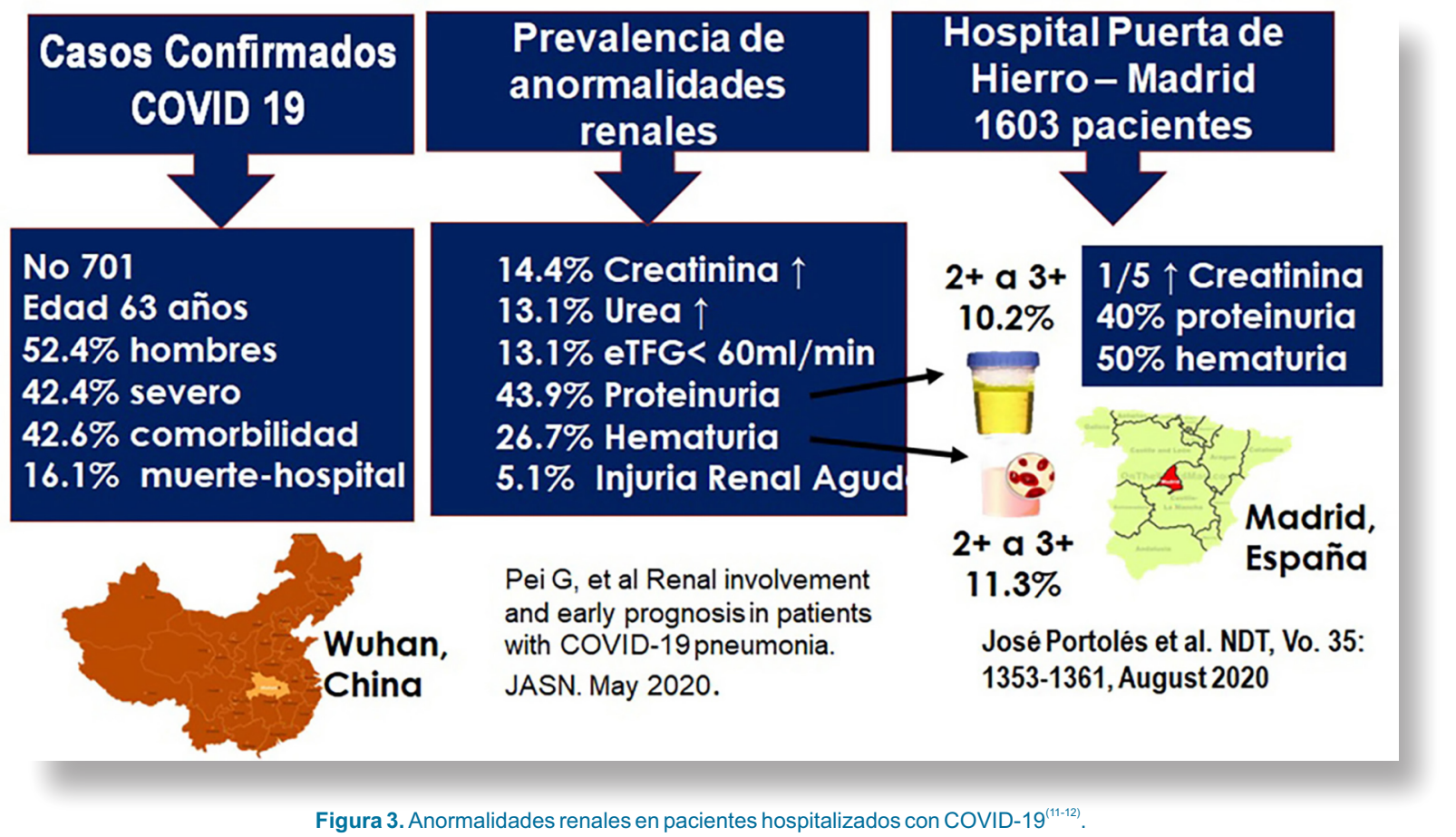

Injuria Renal Aguda (IRA)

La injuria renal aguda (IRA) es una complicación descrita en pacientes con COVID-19, en un estudio realizado en la ciudad de Nueva York, se revisaron las historias clínicas de 5,449 pacientes hospitalizados entre marzo 1 y abril 5. Los estudios fueron hechos en un total de 13 hospitales, entre académicos y comunitarios del área metropolitana de Nueva York. Período de tiempo que involucró a la situación más álgida de la pandemia en la ciudad. Del total de pacientes admitidos 1,993 desarrollaron IRA, representando el 36.6\% de los pacientes, $14.3 \%$ de estos pacientes requirieron terapia de remplazo renal (diálisis). El diagnóstico de IRA se hizo basado en los criterios establecidos por KDIGO. La IRA fue clasificada en tres estadios y con porcentajes de pacientes con IRA: Estadio 1, 46.5\%, estadio $2,22.4 \%$ y estadio $3,31.3 \%$. La IRA fue hallada primariamente en pacientes con falla respiratoria, el $89.7 \%$ de pacientes con asistencia ventilatoria desarrollaron IRA, comparado con $21.7 \%$ en pacientes sin soporte ventilatorio. El $52.2 \%$ de pacientes con IRA, tuvo su inicio dentro de las primeras 24 horas de haber sido intubados. De un total de 285 pacientes que requirieron terapia de diálisis, 276 pacientes estuvieron con ventilación mecánica. Se pudo ver que los factores de riesgo para el desarrollo de IRA incluyeron: edad avanzada, diabetes mellitus, enfermedad cardiovascular, raza negra, hipertensión y la necesidad de ventilación mecánica y uso de vasopresores. Del total de pacientes con IRA, 694 fallecieron (35\%), $519(26 \%)$ fueron dados de alta y $780(39 \%)$ permanecían hospitalizados. En conclusión, de acuerdo a este estudio, la IRA ocurre de manera temprana y en asociación temporal a la falla respiratoria y connota un pronóstico grave (19). (Figura 4).

En un estudio similar, realizado en el Centro Médico Oschner en la ciudad de Nueva Orleans, durante un período de un mes, se encontraron 701 pacientes admitidos al hospital con COVID-19, 70\% de los pacientes fueron negros, 30\% de los pacientes fueron admitidos a una unidad de cuidados intensivos (UCI), y se estableció el diagnóstico de IRA en $28 \%$ de los pacientes (61\% de la UCI). El 35\% de los pacientes mostraron enfermedad renal crónica pre existente. En los pacientes con IRA, se necesitó terapia dialítica en 89 (55\%) y se observó una mortalidad hospitalaria del $55 \%{ }^{(20)}$.

Los estudios realizados en China han mostrado una incidencia de IRA más baja (5\%), comparada con la de los Estados Unidos, una posible explicación estaría relacionada a una presencia más alta de comorbilidades en pacientes de los Estados Unidos comparados con los grupos de pacientes en China ${ }^{(21)}$.

Los estudios descritos han demostrado que el inicio de la IRA tiene una relación temporal a la falla respiratoria y al inicio de ventilación mecánica. Además del proceso séptico y respuesta inflamatoria exagerada en COVID-19, se sabe que el 


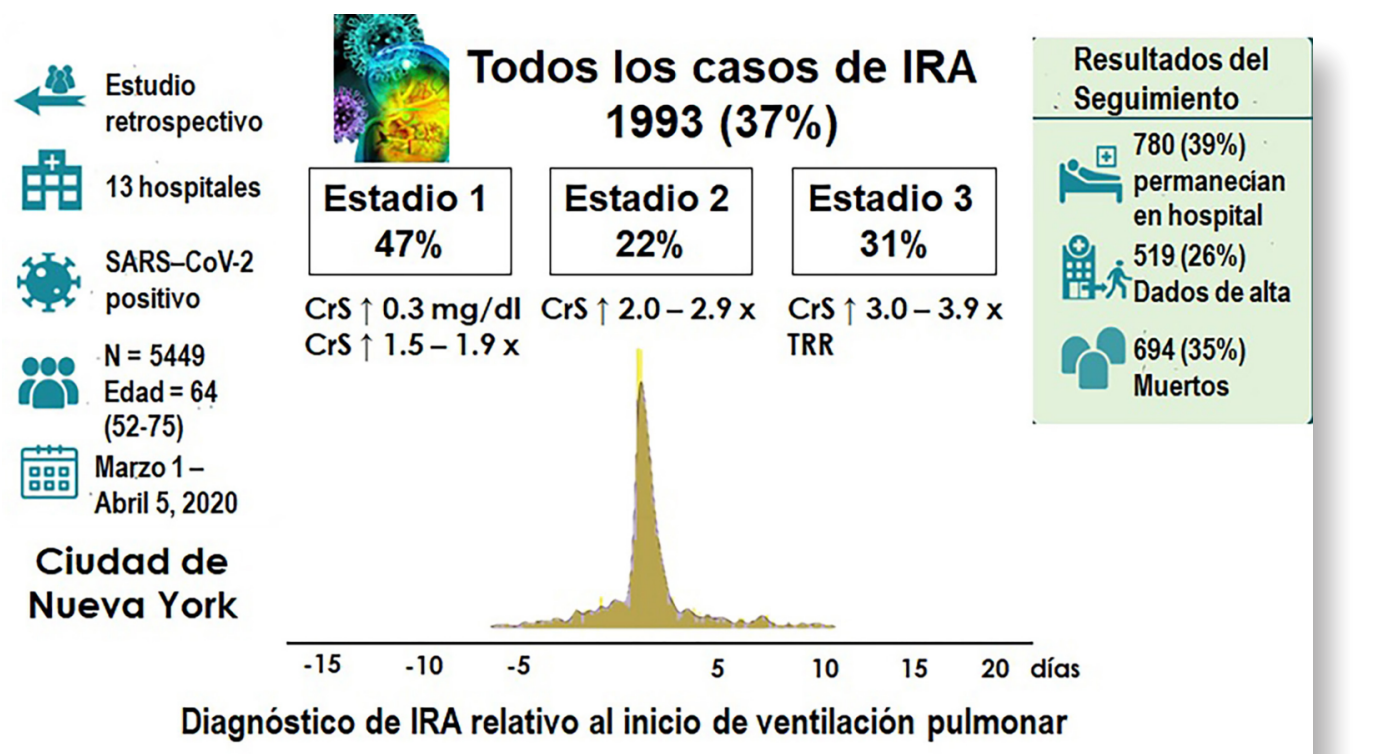

Hirsch JS, et al AKI in patients hospitalized with COVID19. Kidney International Published online May 16, 2020

Figura 4. IRA en pacientes con COVID-19 y su relación al inicio de ventilación pulmonar. Estudio realizado en 13 hospitales de la ciudad de Nueva York ${ }^{(19)}$.

síndrome de distrés respiratorio del adulto (SDRA) es un factor independiente para el desarrollo de IRA ${ }^{(22-24)}$. La ventilación mecánica, por si misma, causa alteraciones fisiológicas con reducción de la precarga cardíaca, la cual conlleva a la disminución del gasto cardíaco, incremento de la presión intratorácica, disfunción ventricular derecha, activación neurohormonal con cambios hemodinámicos que afectan el flujo sanguíneo renal, la presencia de barotrauma incrementa la liberación de citoquinas pro inflamatorias ${ }^{(22)}$. La presencia de hipoxemia, hipercapnia y acidemia asociada al SDRA, también activan el sistema simpático con subsecuente reducción del flujo sanguíneo renal y disminución de la tasa de filtración glomerular. La presencia de hipoxia e hipercapnia inducen vasoconstricción arterial pulmonar, hipertensión y congestión venosa, los cuales constituyen factores agravantes para la función renal ${ }^{(23)}$. La presencia de IRA, agrava al SDRA (Figura $5)$.

\section{Patología Renal en la IRA}

En los estudios de patología renal en pacientes fallecidos con COVID-19 e IRA, realizados en China, los exámenes de microscopía de luz demostraron la presencia de necrosis tubular aguda, con agregados de glóbulos rojos en capilares tubulares. En la microscopía electrónica se describieron partículas virales en podocitos y células epiteliales del túbulo proximal ${ }^{(25)}$. En otro reporte reciente, Farkash ${ }^{(26)}$ nuevamente describe partículas virales en el citoplasma de células epiteliales del túbulo proximal; sin embargo, se ha advertido que dichas imágenes pueden ser confundidas con la presencia de vacuolas intracitoplasmáticas cubiertas de clatrina $(27,28)$.

Dos estudios adicionales de patología renal en pacientes con COVID-19 e IRA de la ciudad de Nueva York, con un total de 22 pacientes, las lesiones patológicas predominantes fueron la de necrosis tubular aguda, no se encontraron partículas virales con exámenes de hibridación in situ para el virus SARSCoV-2 y por exámenes histoquímicos ${ }^{(29,30)}$. En la serie de biopsias postmorten del hospital Lenox Hill de Nueva York ${ }^{(29)}$, se describieron vesículas agregadas cubiertas de clatrina, localizadas en el citosol y con dimensiones variables. En la segunda serie de 10 pacientes de Nueva York ${ }^{(29)}$, se encontraron dos pacientes con hallazgos de microangiopatía trombótica, uno con glomerulonefritis pauci inmune y uno con glomerulopatía colapsante.

Basado en las series de pacientes con COVID-19 e IRA, la patología renal, revela que la patogénesis más frecuente de la IRA es la del desarrollo de necrosis tubular aguda, de probable causa multifactorial: sepsis, hipovolemia, uso de fármacos nefrotóxicos, neumonía, ventilación pulmonar y disfunción cardíaca. 


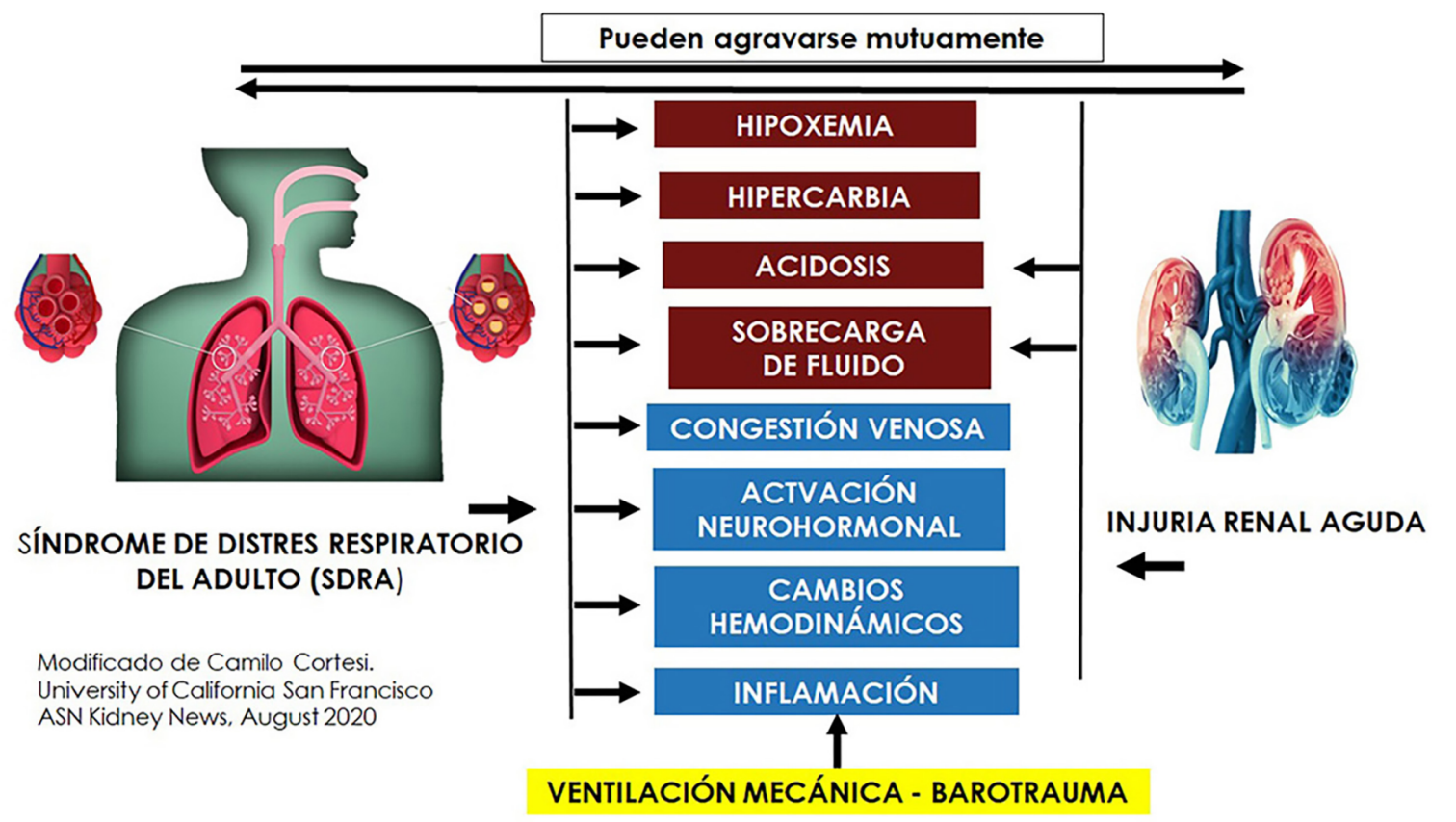

Figura 5. Interacción entre el SDRA, IRAy ventilación mecánica ${ }^{(22)}$

\section{Manejo Clínico de la IRA}

La demanda súbita de servicios hospitalarios de pacientes con COVID-19, muchos de ellos con estadios severos y críticos de la enfermedad, ha requerido de esfuerzos extraordinarios en el personal médico, así como la necesidad de poner en servicio un mayor número de camas de UCI y de equipos de terapia de remplazo renal (TRR). La División de Nefrología del Hospital de la Escuela de Medicina de la Universidad Columbia de la ciudad de Nueva York, entre el 24 de marzo y el 17 de abril, tuvo que potenciar sus servicios de nefrología clínica de 6 a 9 servicios, casi triplicar el número de camas UCI, de 132 a 312, incrementaron el número de procedimientos de hemodiálisis al lado de la cama de 170 a 376, y los procedimientos de terapia de remplazo renal continua de menos de 24 a 67 (pico), también se implementó diálisis peritoneal aguda en 7 pacientes $^{(31)}$.

La incidencia de IRA, reportada en series de pacientes con COVID-19 en Europa y los Estados Unidos, está entre 20 a $40 \%$. De acuerdo al reporte del sistema de salud Northwell de la ciudad de Nueva York $^{(18)}$, la incidencia de IRA fue de 37\%, de estos pacientes el 14.3\% requirieron terapia de diálisis. De pacientes intubados, el $86 \%$ desarrollaron IRA y $23 \%$ de pacientes intubados requirieron diálisis (TRR).

Hasta el momento no existen datos reportados acerca de la incidencia de IRA en pacientes hospitalizados con COVID-
19 en el Perú. Con alrededor de 1400 pacientes que requerían soporte ventilatorio en UCI en el Perú, se estimaría un número significante de pacientes con IRA y que requerirían terapia de diálisis, servicios que no necesariamente estén disponibles en muchas áreas.

Para el manejo terapéutico de pacientes con COVID-19 e IRA, se ha sugerido una guía clínica ${ }^{(32)}$ :

- Maximizar el tratamiento conservador, con especial atención al balance hidro-electrolítico y ácido-base

-Empleo de guías de KDIGO para pacientes críticamente enfermos y a riesgo de IRA ${ }^{(33)}$

-Empleo de furosemida intravenosa

(preferencialmente continua), que permita producir balance de fluido negativo en pacientes con congestión pulmonar, induciendo mejor oxigenación

-En pacientes con COVID-19, hemodinámicamente inestables, y que requieran diálisis, se recomienda terapia de remplazo renal continua(TRRC)

- Terapia de diálisis peritoneal aguda, es una opción válida para pacientes que requieran diálisis, particularmente en centros hospitalarios que no tengan acceso a equipos de hemodiálisis ${ }^{(34)}$. 
En el contexto de iniciación de diálisis peritoneal aguda en pacientes que requieran terapia dialítica de urgencia en medio de la pandemia, es importante puntualizar que pacientes en terapia crónica de diálisis, los cuales en su gran mayoría acuden a centros de hemodiálisis, se encuentran con un incremento de tres veces más de riesgo de contraer COVID-19, comparado con pacientes con terapia de diálisis en el hogar, según datos de la red renal de Ontario, Canadá ${ }^{(35)}$. Una razón más para redoblar los esfuerzos para incrementar la proporción de diálisis peritoneal ambulatoria y hemodiálisis en el hogar.

\section{Referencias bibliográficas}

1. Fischetti M, Hays VF. Glausinger B. Christiansen J. Inside the coronavirus. Scientific American. 2020;323(1)32-37.

2. Wan Y, Shang J, Graham R, Baric RS, Li F. Receptor recognition by the novel coronavirus from Wuhan: an analysis based on decade-long structural studies of SARS coronavirus. J Virol 2020; 947:e0127-20.

3. Guo T, Fan Y, Chen M, et al. Cardiovascular implications of fatal outcomes of patients with coronavirus disease 2019 (COVID-19). JAMA Cardiol 2020.e201017.

4. Rhian M. Touyz, Augusto C. Montezano. Angiotensin-(1-7) and Vascular Function, the Clinical Context. Hypertension. 2018;71:68-69.

5. Aleksova A. et al. COVID-19 and renin-angiotensin system inhibition: role of angiotensin converting enzyme 2 (ACE2) - Is there any scientific evidence for controversy? J Intern Med. 2020 Jun 8: 10.1111/joim.13101.doi: 10.1111/joim.13101 [Epub ahead of print].

6. Mancia G, Rea F, Ludergnani M, Apolone G, Corrao G. Renin-angiotensin-aldosterone system blockers and the risk of COVID-19. N Engl J Med. 2020;382(25):2431-2440.

7. Raisi-Estabragh Z, McCracken C, Ardissino M, Bethell M. et al. Renin-Angiotensin-Aldosterone System Blockers Are Not Associated with Coronavirus Disease 2019 (COVID-19) Hospitalization: Study of 1,439 UK Biobank Cases. Front Cardiovasc Med. 14 July 2020 https://doi.org/10.3389/fcvm. 2020.00138

8. Wysocki J, Lores E, Minghao Y, Soler MJ, Batlle D. Kidney and lung expression after an ACE inhibitor or an ang II receptor blocker: Implications for COVID-19. J Am Soc Nephrol 2020;31:1941-1943.

9. Baral R, White M, Vassiliou V. Effect of renin-angiotensinaldosterone system inhibitors in patients with COVID-19: A systematic review and meta-analysis of 28,872 Patients. Curr Atheroscler Reports. 2020;22:61.

10. Batlle D, Soler MJ, Sparks M, Hiremath S, et al. Acute kidney injury in COVID-19: Emerging evidence of distinct pathophysiology. J Am Soc Nephrol. 2020:31(7):1380-1383.

11. Pei Y, Zhang Z, Peng J, et al. Renal involvement and early prognosis in patients with COVID-19 Pneumonia. J Am Soc Nephrol 2020;31:1157-1165.

12. Portoles J, Marques $\mathbf{M}$, Lopez $\mathbf{P}$, et al. Chronic kidney disease and acute kidney injury in the COVID-19 Spanish outbreak. Nephrol Dial Transplant. 2020;35:1353-1361.

13. Libby $\mathbf{P}$, Lüscher T. COVID-19 is, in the end, an endothelial disease. European Heart Journal; 2020;41:3038-3044.

14. Meijers B, Hillbrands LB. The clinical characteristics of coronavirus associated nephropathy. Nephrol Dial Transplant. 2020;35:1279-1281.

15. Friedman D, Pollak M. Apolipoprotein L1 and kidney disease in African Americans. Trends Endocrinol Metab. 2016; 27(4):204-215.
16. Hotchkkis RS, Monneret G, Payen D. Sepsis-induced immunosuppression: from cellular dysfunctions to immunotherapy Nature Reviews Immunology. 2913;13:862-887.

17. Wu H, Larsen C, Hernandez-Arroyo C, Mohamed M, et al. AKI and Collapsing Glomerulopathy with COVID-19 and APOL1 High-Risk Genotype. J Am Soc Nephrol 2020;31: 1688-1695.

18. Wiersinga WJ, Rhodes A, Cheng A, Peacock S, Prescott H. Pathophysiology, transmission, diagnosis of coronavirus disease (COVID-19). JAMA. 2020;324(8):782-793.

19. Hirsch JS, Ng JH, Ross DW, Sharma P, et al. Acute kidney injury in patients hospitalized with COVID-19. Kidney International. 2020;98:209-218.

20. Mohamed M, Lukitsch I, Torres-Ortiz A, Walker J, et al. Acute kidney injury associated with coronavirus disease 2019 in urban New Orleans. Kidney 360. 2020;1(7):614-622.

21. Chan L. Coca S. Acute kidney injury in the time of COVID-19. Kidney 360. 2020;1(7):588-590.

22.Darmon M, et al. Acute respiratory distress syndrome and risk of AKI among critically ill patients. Clin J Am Soc Nephrol 2014;9:1347-1353.

23. Cortesi C. Acute respiratory distress syndrome from a kidney perspective. US Kidney News. August 2020;12 (8):22-23.

24. Kuiper JW, et al. Mechanical ventilation and acute renal failure. Crit Care Med. 2005;33:1408-1415.

25.Su H, Yang M, Wan C, Yi C, et al. Renal histopathological analysis of 26 postmortem findings of patients with COVID-19 in China. Kidney Int. 2020;98(1):219-227.

26. Farkash EA, Wilson AM, Jentzen JM. Ultrastructural evidence for direct renal infection with SARS-CoV-2. JAm Soc Nephrol. 2020;31(9):2223-2224.

27. Miller S, Brealey J. Visualization of putative coronavirus in the kidney. Kidney Int. July 2020;98 (1):231-232.

28. Miller S, Goldsmith C. Caution in identifying coronavirus by Electron Microscopy. J Am Soc Nephrol. 2020;31(9):22232224.

29. Kudose S, Batal I, Santoriello D, Xu K, et al. Kidney biopsy findings in patients with COVID-19. J Am Soc Nephrol. 2020; 31(9):1959-1968.

30. Sharma P, Uppal N, Wanchoo R, Shah H, et al. COVID-19 Associated kidney injury: A Case series. J Am Soc Nephrol. 2020;31(9):1948-1968.

31. Disaster Response to the COVID-19 Pandemic for Patients with Kidney Disease in New York City. The Division of Nephrology, Columbia University. J Am Soc Nephrol. July 2020;31(7)1371-1379.

32. Ronco C, et al. Management of Acute Kidney Injury in Patients with COVID-19. The Lancet. 2020;8:738-742.

33. KDIGO Clinical Practice Guideline for Acute Kidney Injury. Kidney Int. Supplements. March 2012; Volume 2 (Issue 1). 
34. EI Shamy O, Patel N, Abdelbaset MH, Chenet L1928. Acute start peritoneal dialysis during the COVID-19 Pandemic. J Am Soc Nephrol. 2020;31(8):1680-1682.
35. Brown E, Perl J. Increasing peritoneal dialysis use in response to the COVID-19 pandemic: Will it go viral? J Am Soc Nephrol. 2020;31(9):1928-1930.

Contribución de autoría: Víctor Vladimiro Rozas-Olivera ha sido el autor del estudio, contribuyendo con su concepción, búsqueda electrónica, revisión inicial, el diseño de estudio, redacción y revisión final. Conflicto de interés: El autor no tiene conflictos de interés con la publicación de este trabajo.

Financiamiento: Autofinanciado.

Citar como: Rozas-Olivera, VV. Complicaciones renales secundarias a COVID-19. Diagnóstico(Lima). 2020;59(3):125-132

DOI: $10.33734 /$ diagnostico.v59i3.235

Correspondencia: Victor Vladimiro Rozas Olivera. Correo electrónico: rozasvictor44@gmail.com

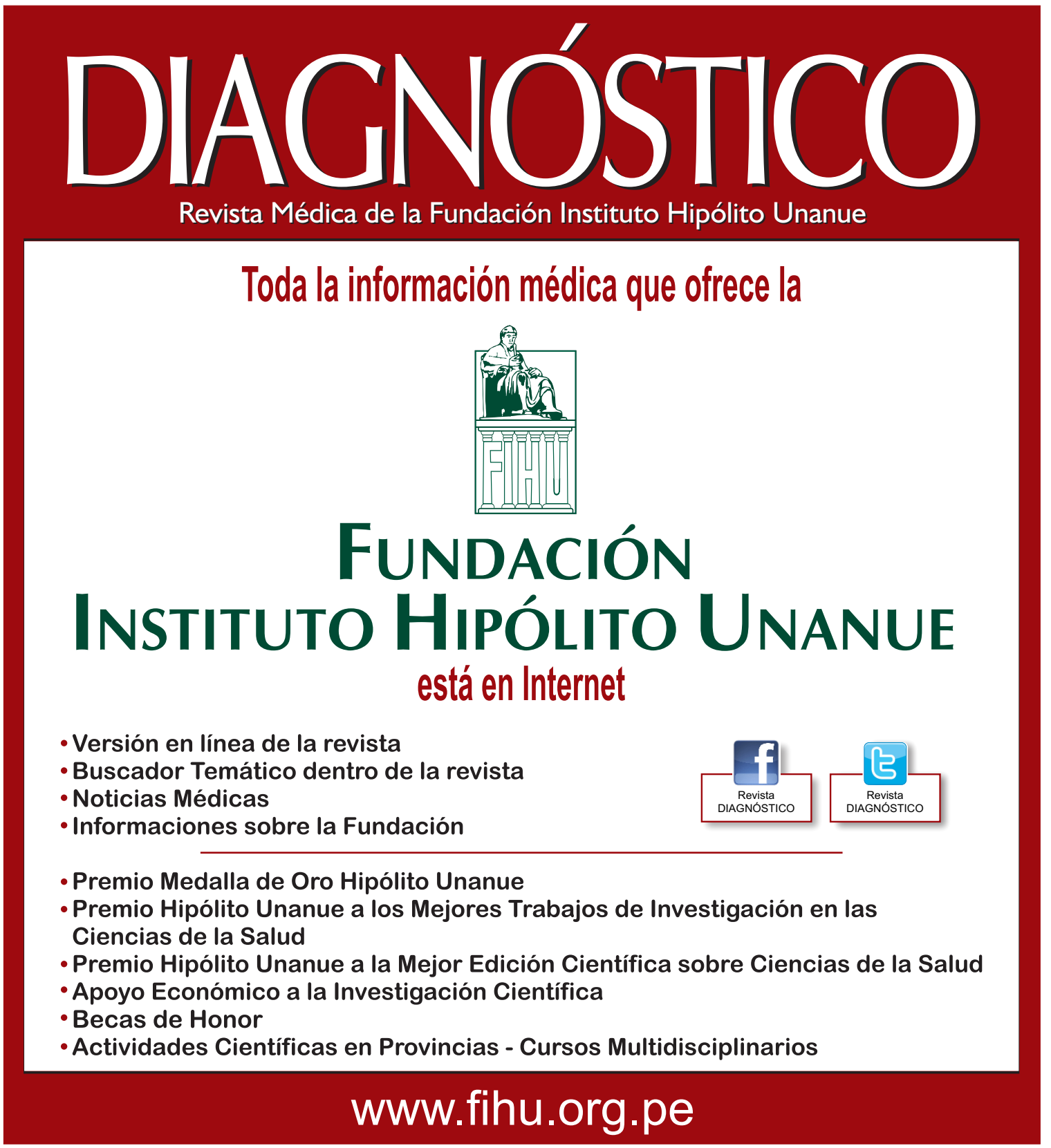

